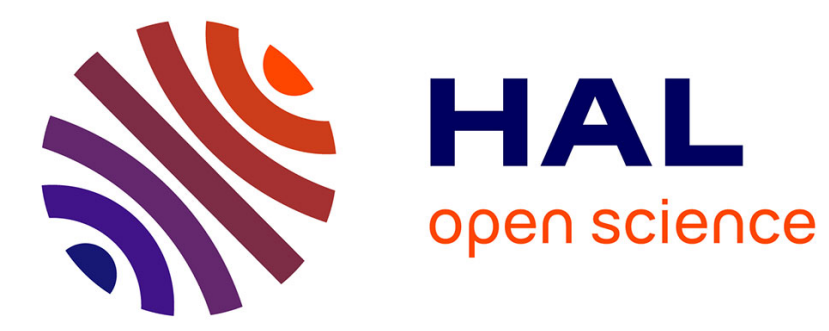

\title{
A unified broadcast layer for horizon 2020 delivery of multimedia services
}

Matthieu Crussière, Catherine Douillard, Christian Gallard, Marie Le Bot, Benjamin Ros, Arnaud Bouttier, Alain Untersee

\section{- To cite this version:}

Matthieu Crussière, Catherine Douillard, Christian Gallard, Marie Le Bot, Benjamin Ros, et al.. A unified broadcast layer for horizon 2020 delivery of multimedia services. IEEE Transactions on Broadcasting, 2014, 60 (2), pp.193-207. 10.1109/TBC.2014.2315764 . hal-01015131

\author{
HAL Id: hal-01015131 \\ https://hal.science/hal-01015131
}

Submitted on 17 Feb 2020

HAL is a multi-disciplinary open access archive for the deposit and dissemination of scientific research documents, whether they are published or not. The documents may come from teaching and research institutions in France or abroad, or from public or private research centers.
L'archive ouverte pluridisciplinaire HAL, est destinée au dépôt et à la diffusion de documents scientifiques de niveau recherche, publiés ou non, émanant des établissements d'enseignement et de recherche français ou étrangers, des laboratoires publics ou privés. 


\title{
“A UNIFIED BRoAdCAST LAYER FOR HORIZON 2020 DELIVERY OF MULTIMEDIA SERVICES", IEEE \\ A Unified Broadcast Layer for Horizon 2020 Delivery of Multimedia Services
}

\author{
Matthieu Crussière, Catherine Douillard, Christian Gallard, Marie Le Bot, Benjamin Ros, \\ Arnaud Bouttier, and Alain Untersee
}

\begin{abstract}
It is expected that, in the coming years, the video data traffic carried by mobile networks will increase drastically. Among the different ways of facing this "mobile data tsunami", the cooperation of several complementary access technologies offers promising prospects. In the context of such a cooperative approach, this paper proposes the definition of a unified broadcast layer, close to the 3GPP LTE technology, and therefore liable to be easily integrated in mobile devices and infrastructure equipment. The general technical requirements for the design of such a common physical layer are detailed and a practical example based on the 3GPP LTE/E-MBMS and DVB-T2 standards is described. The verification of the proposed approach is performed through simulation results and validation tests carried out on a hardware platform.
\end{abstract}

Index Terms-access network cooperation, broadband networks, broadcast networks, common physical layer, DVBT2, hardware demonstrator, satellite channels, terrestrial channels, 3GPP LTE/E-MBMS.

\section{INTRODUCTION}

${ }^{66} \mathrm{G}$ lobal mobile data traffic will increase 13-fold Jetween 2012 and 2017". "Two thirds of the world's mobile data traffic will be video by 2017 ".

These words extracted from [1] and often referred to as the so-called "mobile data tsunami" describe how strong the phenomenon of data traffic growth is expected to be in the coming years. With such figures in mind, one can question how mobile operators will be able to face this challenge.

The evolution in network topologies, from current macro-cells to future small cells (picocells, femtocells), will provide more capacity locally. The counterparts of such a network densification are twofold. On the one hand, interference will increase and will have to be managed in a not too complex way; technical solutions are currently being studied in the 3GPP standardization groups. On the other hand, backhauling issues will also have to be solved: how to connect so many small cells to the core network, in a costefficient way, while guaranteeing the desired quality of experience for the end user?

In order to provide higher throughput services, a mobile operator can also acquire more spectrum, as data rates are

This work was carried out in the framework of the French collaborative project Mobile MultiMedia $\left(\mathrm{M}^{3}\right)$, funded by the French National Research Agency, project ANR-2010-VERS-0010.

Matthieu Crussière is with the INSA-IETR Laboratory, Rennes, France. Catherine Douillard is with the Telecom Bretagne/Lab-STICC Laboratory, Brest, France. Christian Gallard and Marie Le Bot are with Orange Labs, Rennes, France. Arnaud Bouttier is with Mitsubishi Electric R\&D Centre Europe, Rennes, France. Alain Untersee is with Teamcast, Rennes, France. Benjamin Ros is with CNES, Toulouse, France. closely related to the amount of available spectrum. To this end, the digital dividend in the $800 \mathrm{MHz}$ band has already been allocated to mobile operators and the $700 \mathrm{MHz}$ band allocation will be discussed during the World Radiocommunication Conference in 2015. Consequently, one can imagine that the spectrum size allocated to mobile operators could be roughly doubled by 2020 . Nevertheless, such investments in spectrum have a non-negligible cost for mobile operators. A longer term perspective, in terms of spectral resources, could be the use of millimeter waves where huge bandwidths could be available for small cells, due to the short coverage capability at such high frequencies. For instance, the $60 \mathrm{GHz}$ band has been identified as a promising band: a huge amount of unlicensed spectrum is available and acceptable attenuation levels are observed for these frequencies.

Another alternative is to rely on the cooperation of different access technologies. One current example of such cooperation involves offloading part of the mobile traffic over WiFi access networks: in indoor environments such as homes, offices, or airports, the mobile traffic can be diverted to private or public WiFi access points when available. In order to improve the cooperation, enhancements are about to be carried out in 3GPP LTE (Long Term Evolution), release 12, and WiFi standards (known as High Efficiency WiFi [2]).

A well-known technology which can be also very helpful in delivering video services is broadcasting. Indeed, broadcasting should not be restricted to live TV delivery. It can also beneficially provide other kinds of multimedia services in the coverage area of the network and can then be considered as an efficient "point-to-area" service distribution technology. In particular, taking advantage of the huge amount of memory available in devices, broadcasting is well suited for predictive datacasting services. Different types of contents, such as e-magazines, "top-10" music video-clips, software and application updates, etc., can be pushed to device cache prior to their consumption by the end user, who can then access the contents, even without any connection, with a perfect quality of service. Besides, such a service based on broadcast access technology and known as NOTTV [3] is already available in Japan since April 2012.

In the framework of the DVB-NGH (Digital Video Broadcasting-Next Generation Handheld) standardization process, it was proposed in February 2010 to re-use 3GPP E-MBMS (Evolved Multimedia Broadcast Multicast Services), i.e., the $4 \mathrm{G}$ broadcast mode, as a basis for $\mathrm{NGH}$ specifications. The purpose of this proposal was to avoid market fragmentation between standards, while making it 
easier the integration of broadcast chipsets in mobile devices (e.g., smartphones and tablets).

However, 3GPP E-MBMS as currently specified in Release 9 [4] is not able to meet all the usual broadcasters' requirements. For instance, terrestrial broadcast networks can usually cover areas within a radius of several tens of kilometres. In contrast, E-MBMS signals transmitted in a shared-carrier mode with LTE unicast data can only reach users at a maximum distance of $5 \mathrm{~km}$ from the base station, if no specific processing is performed on the receiver side. Moreover, as latency is not an issue for broadcasting services, deep time interleaving can be applied in such systems, which leads to improved performance in terms of quality of service, particularly in mobile conditions. For the development of the LTE standard, one of the initial requirements was conversely the reduction of latency compared to $3 \mathrm{G}$; so no specific time interleaver has been designed for the LTE broadcast mode.

In November 2010, the DVB Forum decided to knock at 3GPP's door in order to propose a common definition for a unified broadcast standard. After an initial phase of mutual presentations of DVB and 3GPP broadcast state-of-the-art standards, a small group of 3GPP companies decided to propose, in May 2011, the creation of a study item dealing with converged mobile broadcast support for LTE. Unfortunately, due to the lack of support from mobile key players (due to unclear business model, uncertain potential profit for operators, etc.), the study item creation proposal was rejected in the end.

The various past attempts to propose pure broadcast solutions to the mobile ecosystem turned out to be vain. DVB-H, the first generation of DVB broadcast standard to handhelds standardized in 2004 [5], which was promoted by Nokia, has met a rather mixed success. DVB-H chipsets have been integrated into Nokia handsets, some services over DVB-H networks have been launched, e.g., in Finland, Austria, and Italy, but most of these services have now been switched off. On the other side of the Atlantic, Qualcomm promoted a proprietary solution known as MediaFLO [6] and got this technology standardized by the European Telecommunications Standards Institute (ETSI) in 2009 [7]. FLO TV services have been launched in the US starting from 2007. Some devices able to receive such services were introduced by LG and Samsung in 2006. Nevertheless, in 2010 Qualcomm decided not to support the MediaFLO technology any longer and sold the spectrum they owned in the $700 \mathrm{MHz}$ band to AT\&T. A second trial on the DVB side with DVB-SH (Satellite Services to Handhelds) [8], mainly promoted by Alcatel Lucent, did not meet the expected commercial success either.

A few lessons can then be learnt from these experiences:

- A mobile broadcast standard would more likely become a market success if supported by a worldwide ecosystem, e.g. by 3 GPP actors. Without having mobile vendors in the loop from day one, convincing them to embed a non3GPP broadcast chipset in their devices afterwards seems to be difficult.

- Deploying and managing a broadcast network in addition to a mobile network also seems hard to achieve. This is mainly due to cost issues since significant investments have to be made to deploy this second network.
Beyond historical broadcast services (live TV and radio), additional multimedia services must be considered in order to assess the actual interest in deploying a broadcast solution.

The original approach described in this paper, consists in defining a universal broadcast layer, close to the LTE technology, and therefore liable to be easily integrated in mobile devices and infrastructure equipment, which is an essential enabling factor for a worldwide adoption.

This paper is organized as follows. Section II analyses the general requirements for a common physical layer (CPHY) and compares the main features of the DVB-T2/NGH standard with the broadcast mode of 3GPP LTE. A set of CPHY specifications based on LTE/E-MBMS is then proposed in section III. Section IV describes a hardware demonstrator implementing the proposed CPHY and presents the different validation tests carried out so far through simulations and laboratory tests. Section V concludes the paper.

\section{FOUNDATIONS FOR A COMMON PHYSICAL LAYER}

In this section, we introduce the concept of CPHY specifications for a convergent broadband/broadcast network. The technical challenges to obtain such a unified system are first detailed from a general point of view according to the specific requirements of point-to-area transmissions. Then, a comparison between the broadcast systems coming from DVB and 3GPP standardization bodies is carried out to better understand the key features that should govern the technical choices for a future common broadcast physical layer.

\section{A. Common Physical Layer Challenges}

The CPHY specifications have to be devised to act as an enabler for the deployment of broadcasting transmission modes over various network infrastructures, whether it be conventional terrestrial broadcast, broadband networks or even satellite ones. Such a unified system should allow a common baseband chip embedded in a handheld receiver to demodulate and decode any service delivered through a broadcast transmission mode whatever the bearer network responsible of the transmission. In other words, baseband operations for broadcast reception should become network agnostic. In this perspective, many basic features have to be guaranteed to make the CPHY well suited for point-to-area transmissions whatever the network environment, i.e., whatever the point (the transmit station) and whatever the area (the radio links).

At first, the CPHY waveform should be sufficiently scalable to be fully compatible with the radio deployment planning of the targeted networks in terms of frequency bands, coverage areas and frequency planning strategies. For instance, the CPHY has to be able to address at once typical frequency bandwidths for mobile communications and Digital TV (DTV). Moreover, as the cell range of cellular networks is far different from the coverage areas targeted by terrestrial or even satellite DTV networks, the CPHY waveform has to be shaped in order to ensure the coverage of cells whose size ranges from a few hundred meters to several tens of kilometers. This is of particular importance when considering Single Frequency Network (SFN) planning for which the coverage distance is mainly driven 
by the waveform immunity against inter-cellular interference.

Secondly, CPHY should take advantage of the full downlink feature of broadcast transmissions by defining long frame durations. In a pure broadcast mode, transmission frames can indeed be designed without any delay or latency considerations due to the uplink channel constraints. As far as modulation and coding schemes are concerned, using long frames allows the constellations and coding rates to be optimized for large codeword lengths, making the system perform closer to the Shannon limit. Over time-varying channels typical of mobile reception scenarios, interleaving schemes should also be applied over a large time spread to take advantage of time diversity. The increase of the interleaving depth and codeword length should actually essentially be limited by the maximum memory size that can be embedded in nomadic terminals.

Finally, the CPHY specifications should be based on a framing structure adapted to point-to-area transmissions. Such transmissions ensure a universal coverage, i.e., they are not user specific and they deliver a common content to all the terminals within the coverage area whatever their individual situations. This implies that a given user should be able to access the broadcast contents at every time without engaging complex high-level process such as session control protocols. Besides, the user terminal should be able to easily and rapidly extract the multimedia service of interest among the whole delivered content. This involves designing frames with their own embedded signaling messages, giving all the necessary information about the frame structure and service content. Additionally, the frame structure should allow fast synchronization and sounding procedures.

\section{B. Comparison of the DVB-T2/NGH and $3 G P P$ LTE standards}

This section analyses the state-of-the-art terrestrial DVB and 3GPP cellular broadcasting technologies. In Europe, the DVB standardization body proposes specifications for signal transmission over terrestrial, satellite, and cable broadcast networks. More specifically, the DVB-T2 [9] and DVBNGH [10] systems define a family of frames able to operate broadcasting in various network topologies. On the other hand, the 3GPP working groups in charge of LTE have introduced the E-MBMS broadcast mode for cellular network operation [4]. These two systems can thus be viewed as reference systems for this study and the targeted $\mathrm{CPHY}$ has been designed taking their specifications as bases.

The first stage of comparison deals with the framing approach. Fig. 1 and Fig. 2 give the overall frame structure in both systems. DVB-T2 is based on a super-frame time segmentation possibly containing different families of frames whose duration can reach $250 \mathrm{~ms}$. It can be noticed that the DVB-T2 framing provides the concept of Future Extension Frames (FEF) for the transmission of non-DVBT2 frames. For LTE, Fig. 2 gives the framing structure defined for downlink in the Frequency Division Duplex (FDD) mode. In this mode, the LTE radio interface supports radioframes of $10 \mathrm{~ms}$ subdivided into ten sub-frames of $1 \mathrm{~ms}$ each. This sub-frame duration is a reference value for the LTE system and is referred to as Time Transmit Interval
(TTI). As shown in the figure, a subset of the downlink subframes can be configured to deliver E-MBMS broadcast services/signals and E-MBMS services are multiplexed with classical unicast services within the radioframes. Both

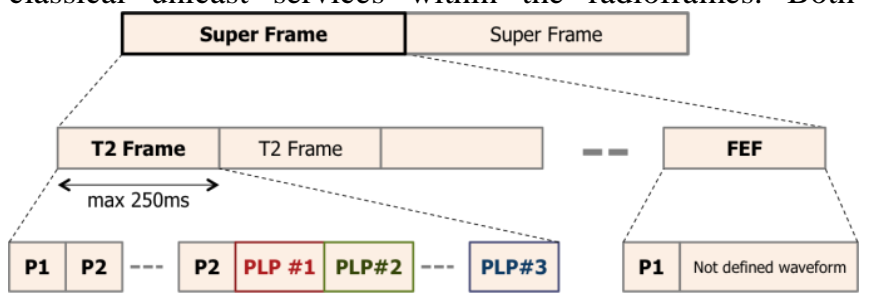

Fig. 1: Schematic view of the DVB-T2 frame structure highlighting P1 and $\mathrm{P} 2$ preambles, multiple physical layer pipes (PLP) multiplexing and future extension frame (FEF) insertion.

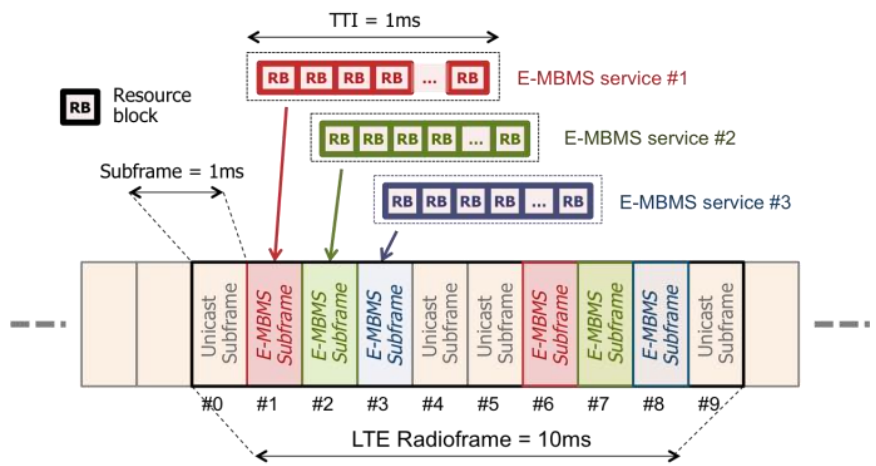

Fig. 2: Schematic view of the LTE radioframe structure (in FDD mode) highlighting the resource block allocation units and showing the unicast and E-MBMS services multiplexing approach.

systems allow several services to be multiplexed within a frame.

This feature is provided by Physical Layer Pipes (PLP) in DVB-T2, each PLP being associated to a given set of physical layer parameters (modulation order, coding rate, interleaving depth) corresponding to a particular quality of service. In LTE, service multiplexing is inherent to the broadband nature of the systems. Each LTE service is first allocated to a set of elementary containers called Resource Blocks (RB) which are mapped onto the subframes. Last but not least, the two systems are based on very different signaling approaches.

On the DVB side, multiplexes of linearly programmed TV services are self-documented (the Electronic Programme Guide gives information on multiplexed services and programmed contents), requiring the receivers to discover the TV services. On that basis, DVB-T2 frames are provided with dedicated preambles, namely P1 and P2 (see Fig. 1), to allow the terminal to be fast synchronized and to easily extract the desired service from the frame payload. On the LTE side, broadband services are intermittently using the transmission resources and then are sustained by session opening/closing procedures implemented through a control channel using the bidirectional wireless connection. Hence, synchronization and signaling information is shared between unicast and broadcast services and is distributed across the whole radioframe.

To go further in the system comparison, Table I gives a summary of the main physical layer characteristics of these two broadcast standards. On the DVB side, DVB-T2 Lite parameters are taken as a reference. They constitute a subset of the DVB-T2 specifications that is well suited for mobile 
TRANS. BROADCASTING, SPECIAL ISSUE ON FOBTV

TABLE I

COMPARISON OF DVB-T2 LITE AND 3GPP E-MBMS PHYSICAL LAYERS

\begin{tabular}{lcc}
\hline \hline & 3GPP E-MBMS & DVB-T2 Lite \\
\hline Frequencies & & \\
Main Frequency bands & {$[791-862]$} & {$[174-446]$} \\
in Europe $[\mathrm{MHz}]$ & {$[2,500-2,690]$} & {$[470-790]$} \\
Main Channel & $\mathbf{5} ; 10 ; 15 ; 20$ & $5 ; 6 ; 7 ; \mathbf{8}$ \\
Bandwidths $[\mathrm{MHz}]$ & & \\
\hline OFDM parameters & Bandwidth $5 \mathrm{MHz}$ & Bandwidth $8 \mathrm{MHz}$ \\
FFT size & From 128 to $2 \mathrm{k}$ & From $2 \mathrm{k}$ to $16 \mathrm{k}$ \\
$\Delta f$ [kHz] & 15 & From 4.464 to 0.558 \\
Symbol duration $[\mu \mathrm{s}]$ & 67 & From 224 to 1792 \\
Guard Interval $[\mu \mathrm{s}]$ & 16.67 & From 14 to 448 \\
Cell radius $[\mathrm{km}]$ & 5 & From 4 to 133 \\
& & \\
\hline Chamnel coding & &
\end{tabular}

\begin{tabular}{lcc}
\hline Channel coding & Binary turbo-code & LDPC code \\
Code family & Variable $K=\{40 \ldots 6144\}$ & Fixed $N=16200$ \\
Codeword size [bits] & From 0.1172 to 0.9258 & From $1 / 3$ to $11 / 15$ \\
Code rate & 1 ms max (TTI length) & 250 ms max \\
Interleav. Depth [ms] &
\end{tabular}

Channel Sounding

$\begin{array}{ll}\text { Scattered pilots } & \text { Fixed pattern (see [4]) } 8 \text { Pilot patterns }\end{array}$

reception. As far as 3GPP is concerned, the LTE E-MBMS downlink parameters have been reported in the table, based on the FDD mode of the LTE standard.

These physical layers have a number of commonalities. Firstly, they use an OFDM modulating waveform, due to the fact that they have both been designed to cope with time and frequency selective channels. Various modulation and coding schemes are available in each standard as well, in order to provide flexible system performance. Such flexibility is exploited at the user level on the LTE side whereas it is viewed on the DVB side as the ability for the operator to select sets of parameters according to the targeted network deployment. Besides, DVB and LTE systems carry out a similar approach for channel sounding, using dedicated pilot symbols spread over the OFDM spectrum according to specific patterns. As a last common feature, both systems define SFN modes. For DVB systems, this is actually a basic feature used for the conventional deployment of a terrestrial network.

Apart from these similarities, both systems differ on various key aspects as highlighted in Table I. First of all, both systems operate in different frequency bands. Terrestrial DVB systems were assigned the VHF and UHF bands whereas LTE systems address bandwidths in the frequency regions around $2.6 \mathrm{GHz}$. Note however, that the $\mathrm{S}$ band between 2 and $4 \mathrm{GHz}$ is envisaged for the future DVBNGH satellite link. In addition, some countries have recently taken the opportunity of the digital dividend to reallocate the upper band of the UHF spectrum to LTE systems, namely between 790 and $862 \mathrm{MHz}$. Concerning bandwidths, typical values for DTV broadcasting are 6,7 and $8 \mathrm{MHz}$ for the terrestrial link and $5 \mathrm{MHz}$ for the satellite link. LTE systems are mainly based on bandwidths defined as multiples of $5 \mathrm{MHz}$. So, it turns out that the two systems are not natively compatible in terms of spectrum usage, which is a first degree of harmonization to deal with (see section III).

As a second major difference, the analysis of the OFDM parameters shows that they have not been chosen following the same strategy. Concerning the LTE standard, the OFDM waveform is governed by a fixed inter-carrier spacing of $15 \mathrm{kHz}$, the desired OFDM spectrum width being actually obtained through the activation of an adequate number of subcarriers at the FFT stage. Such a large inter-carrier spacing value allows strong Doppler effects to be handled. LTE has in fact been designed to support high mobility scenarios in frequency bands than can be located at $2.6 \mathrm{GHz}$. In addition, larger values of the inter-carrier spacing are more convenient to limit the uplink synchronization constraints. As a consequence of this choice, the time domain OFDM symbol length remains constant, namely $67 \mu$ s, whatever the effective signal bandwidth. Hence, considering a maximum ratio of $1 / 4$, the guard interval duration is restricted to $16.67 \mu \mathrm{s}$. On the other hand, the DVB OFDM parameters result from a trade-off between Doppler effect mitigation and guard interval increase. Actually, DVB offers several FFT sizes for each of the possible bandwidths. This leads to variable inter-carrier spacing values and thus to a variable degree of resistance against Doppler effect. Besides, this gives rise to various symbol and guard interval durations. From Table I, we can note for instance that the inter-carrier spacing for an $8 \mathrm{MHz}$ bandwidth ranges from $0.558 \mathrm{kHz}$ to $4.464 \mathrm{kHz}$, yielding guard interval values between $14 \mu$ s and $448 \mu$ s. The idea behind the guard interval adjustment is to adapt the OFDM signal to various coverage ranges if the system is operated in SFN mode. In such a network configuration, the guard interval is chosen according to the distance between two neighboring transmit towers so that it is able to absorb the echoes of the signals coming from each transmission link. In other words, the guard interval duration can directly be translated into maximum coverage distance according to wave propagation speed, as reported in Table I. As an example, a maximum cell radius of $133 \mathrm{~km}$ is given for the DVB system considering an $8 \mathrm{MHz}$ bandwidth, which is far larger than the $5 \mathrm{~km}$ obtained for the LTE standard. However, LTE also specifies an SFN mode allowing the inter-carrier spacing to be reduced to half of its nominal value, i.e., $7.5 \mathrm{kHz}$, and leading to cells with an increased 10 $\mathrm{km}$ radius. This value still remains 13 times smaller than the maximum radius in terrestrial DVB. From this comparison, we can conclude that LTE systems are mainly designed for Doppler effect mitigation, whereas DVB systems are tailored to fit large coverage areas. The search of adequate OFDM parameters for a common broadcast PHY under coverage and mobility constraints is investigated in section III.

The last significant contrast between DVB and LTE specifications can be analyzed from a Forward Error Correction (FEC) coding and interleaving point of view. At a first level, the two systems exploit different families of error correcting codes: DVB is based on LDPC codes while LTE makes use of turbo codes. However, when properly designed, both code families display comparable performance for similar implementation complexity. Consequently, the FEC code family does not represent a key criterion in our analysis. We rather focus on the FEC block size and time interleaving duration values. On the DVB side, the coded block size is fixed and is equal to 16,200 bits; the 

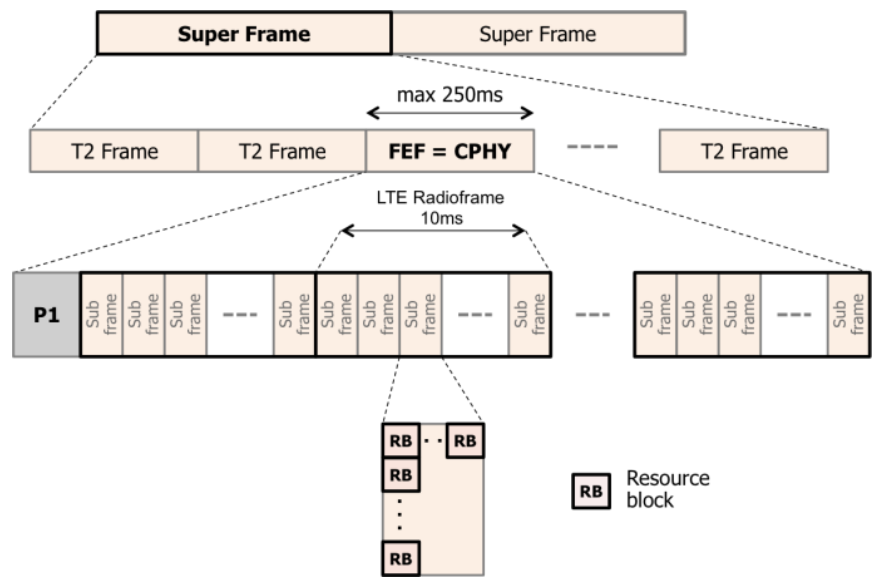

Fig. 3: CPHY framing structure proposal as a particular DVB-T2 FEF. The resource allocation principles of $3 \mathrm{GPP}$ LTE, based on resource block units and sub-frame segmentation, are maintained.

information block size thus depends on the coding rate. Then, bit interleaving is carried out at the FEC block level and time interleaving is applied to the constellation symbols within the so-called interleaving frames, which can contain a variable number of FEC blocks. As a result, the overall interleaving depth can reach the maximum frame duration, i.e., $250 \mathrm{~ms}$ (see Table I and Fig. 1). It can even be extended to larger values with the inter-frame interleaving option which allows each interleaving frame to be mapped across several T2 frames. In this case, the improvement of the interleaving depth comes at the price of a bit-rate reduction and latency increase and has thus to be decided accordingly.

For the satellite case, which is the most sensitive to time selectivity, an interleaver with a time spread of several seconds could be envisaged [14]. From these considerations, it appears that DVB systems have been designed to benefit from high time/frequency diversity in a full downlink transmission mode. On the 3GPP side, the followed strategy is different. Table I indicates that various information block sizes (before coding) ranging from 40 to 6,144 bits are available. Compared to the DVB specifications, the LTE system thus offers more flexibility with respect to the block size, which can be explained by the need to adapt each link to users' requirements in cellular networks. Consequently, interleaving essentially relies on the interleavers used by the turbo code and on the so-called rate-matching functionalities, thus limiting the interleaving depth to a single code block. Each code block is then allocated to the resource blocks defined as the atomic allocation unit in LTE systems. In E-MBMS with SFN operation, these resource blocks are mapped without any additional interleaving process onto the subframes introduced in Fig. 2. Eventually, the maximum time spread in LTE E-MBMS is limited to the $1 \mathrm{~ms}$ TTI value, which is far lower than what is carried out on the DVB side. The reason for such a difference comes from the fact that the LTE systems have to manage bidirectional communications governed by delay constraints. Nevertheless, LTE FEC functionalities include retransmission procedures that provide the base station with the possibility to transmit additional redundancy in case of erroneous packet reception at the terminal side, hereby implicitly exploiting channel time diversity. In a pure downlink broadcast transmission, such an approach can obviously not be carried out. Hence, in the perspective of a convergent E-MBMS/DVB system, interleaving is a fundamental issue to be addressed.

\section{CPHY SPECIFICATIONS PROPOSAL}

Mobile broadcasting can be viewed as standing at the confluence of the DVB and 3GPP worlds. Aiming at defining a unified physical layer for mobile broadcasting scenarios, it is highly desirable to take advantage of both system designs. Our approach involves starting from the LTE/E-MBMS specifications and making them evolve to integrate broadcasters' requirements in the perspective of a deployment of the CPHY over traditional broadcasting infrastructures, terrestrial and satellite.

Regarding the comparison analysis made in section II, the E-MBMS specifications are modified according to the following steps:

- Define a frame structure compatible with the DVB framing approach;

- Modify the OFDM parameters in order to address typical broadcasters' bandwidths;

- Increase the OFDM guard interval duration for SFN operation at the scale of typical broadcasters' cells;

- Increase time diversity exploitation with time interleaving;

- Design appropriate pilot patterns according to the previous modifications.

\section{A. Framing}

As detailed in section II.B, E-MBMS services are multiplexed with unicast services within the LTE radioframes. The related synchronization and signaling procedures rely on information available in the unicast part of the frame. Since CPHY signals should be delivered through downlink-only transmissions, it should be rather viewed as a dedicated carrier mode from the 3GPP world. The CPHY system has to embed its own synchronization and signaling information. Even if this paper is not intended to give complete solutions about signaling and synchronization mechanisms, some basic concepts are discussed hereafter. A simple solution is for instance to follow the preamble concept offered by the DVB-T2 specifications as previously described and depicted in Fig. 1. A dedicated P1 symbol can be defined to enable quick synchronization and to carry some preliminary signaling. To go further, the CPHY can be considered as a particular FEF and can be multiplexed in time with already existing DVBT2 frames, the dedicated preamble P1 guaranteeing its proper identification. This idea is sketched in Fig. 3. To keep the CPHY system as close as possible to the E-MBMS specifications, the new frames can benefit from the resource allocation strategy defined by 3GPP. In practice, we propose to build CHPY frames from radioframes of $10 \mathrm{~ms}$ made of subframes of fixed duration and relying on the elementary RB containers used by the 3GPP LTE systems. This approach gives a solution to the deployment of CPHY frames either in a TDM fashion with DVB-T2 signals or directly embedded in a LTE radioframe. The practical implementation of a time multiplex with native sampling frequencies of each system (DVB and 3GPP) is discussed in 
section IV.B.

\section{B. Bandwidth harmonization}

As previously mentioned in section II.B, three native bandwidths of the DVB-T2 standard, i.e., $6 \mathrm{MHz}, 7 \mathrm{MHz}$ and $8 \mathrm{MHz}$, are not addressed by current E-MBMS specifications. As a universal system, CPHY should be compatible with these bandwidth values. Reminding that 3GPP LTE is somehow bandwidth agnostic, it is actually fairly simple to adapt E-MBMS signals to these three new bandwidth cases. This is achieved through the activation of the adequate number of subcarriers for a fixed inter-carrier spacing and a given FFT size. Following 3GPP terminology, this translates into the allocation of a variable number of RBs per OFDM symbol, one RB corresponding to $180 \mathrm{kHz}$. As an example, one can easily achieve the 6,7 or $8 \mathrm{MHz}$ bandwidths starting from the existing $10 \mathrm{MHz}$ E-MBMS case by progressively reducing the number of modulated subcarriers. Following this idea, one can calculate the number of subcarriers, or alternatively the number of RBs, being allocated according to the system bandwidth. Table II reports the parameters obtained under the $15 \mathrm{kHz}$ and $7.5 \mathrm{kHz}$ legacy E-MBMS subcarrier spacing and considering 1024- and 2048-point FFTs respectively. Note that a similar approach can be followed starting from a $5 \mathrm{MHz}$ bandwidth with a 512-point FFT and then increasing the number of RBs. The choice between these two possibilities can actually be related to implementation strategies.

It is worth noting that the above proposal keeps approximately the same bandwidth occupancy as in the legacy E-MBMS standard, in such a way that the ratio of effective bandwidth over the total bandwidth is around $90 \%$. Hence, this proposal retains the spectrum usage policy in use on the $3 \mathrm{GPP}$ side and could be considered as a "3GPP compatible" allocation mode. On the DVB side, an extended bandwidth planning is traditionally adopted to increase the system capacity. With this goal in mind, a second bandwidth allocation mode called "DVB compatible" can be considered with improved bandwidth occupancy, yet respecting DVB spectrum usage. The figures for these two modes are reported in Table III, highlighting an improvement of around $5 \%$ of the spectral occupancy when the extended mode is activated.

TABLE II

CPHY PARAMETERS FOR UHF BANDWIDTHS COMPATIBILITY, STARTING FROM THE LTE-NATIVE $10 \mathrm{MHZ}$ BANDWIDTH

\begin{tabular}{cccccc}
\hline \hline $\begin{array}{c}\text { Radio } \\
\text { Bandwidth }\end{array}$ & $\begin{array}{c}\text { Inter-carrier } \\
\text { spacing }\end{array}$ & $\begin{array}{c}\text { FFT } \\
\text { size }\end{array}$ & $\begin{array}{c}\text { Number } \\
\text { of RBs }\end{array}$ & $\begin{array}{c}\text { Activated } \\
\text { subcarriers }\end{array}$ & $\begin{array}{c}\text { Effective } \\
\text { Bandwidth }\end{array}$ \\
\hline \multirow{2}{*}{$10 \mathrm{MHz}$} & $15 \mathrm{kHz}$ & 1024 & 50 & $\begin{array}{c}600 \\
1200\end{array}$ & $9 \mathrm{MHz}$ \\
\hline \multirow{2}{*}{$8 \mathrm{MHz}$} & $7.5 \mathrm{kHz}$ & 2048 & & 480 & $7.2 \mathrm{MHz}$ \\
\hline \multirow{2}{*}{$7 \mathrm{MHz}$} & $7.5 \mathrm{kHz}$ & 2048 & 40 & 960 & \multirow{2}{*}{$6.3 \mathrm{MHz}$} \\
\hline \multirow{2}{*}{$6 \mathrm{MHz}$} & $15 \mathrm{kHz}$ & 1024 & 35 & 840 & \\
& $7.5 \mathrm{kHz}$ & 2048 & & 360 & $5.4 \mathrm{MHz}$ \\
\hline
\end{tabular}


column of Table IV indicating the number of OFDM symbols to map to subframes. Eventually, the smallest value of inter-carrier spacing, namely $1.25 \mathrm{kHz}$, is still compatible with the subframe segmentation and leads to the allocation of a single OFDM symbol per subframe.

\section{Interleaving depth increase}

As explained in section II, the time interleaving depth offered by the 3GPP specifications is significantly smaller than those specified in the DVB-T2/T2-Lite standards, due to latency constraints. Two main strategies detailed hereafter are proposed to circumvent this limitation of the physical layer:

- Intra- and inter-frame interleaving schemes based on enlarged TTI and time slicing concept,

- Retransmission-based interleaving schemes reusing the Hybrid Automatic Repeat reQuest (HARQ) concept.

Note that both approaches can only be carried out if additional soft buffer resources are made available at the terminal.

\section{Inter-frame and intra-frame interleaving}

A simple way to increase the frequency/time diversity of the 3GPP E-MBMS system is to incorporate an interleaving scheme at the Multicast Channel (MCH) level while increasing the TTI value. In current 3GPP systems, an $\mathrm{MCH}$ is mapped onto a single TTI of $1 \mathrm{~ms}$ based on the resource allocation approach depicted in Fig. 2. Keeping the same mechanism, the TTI value could actually be increased to $2 \mathrm{~ms}$ for instance, while adding an intra-frame interleaving applied on the coded blocks mapped onto the RBs. Following this example, data allocation within the frame is simply carried out as described in Fig. 4. This principle can also be extended up to a $5 \mathrm{~ms}$ TTI in a $10 \mathrm{~ms}$ radioframe, or to even larger values if the LTE nominal radioframe duration is extended. Complementary to this, time slicing mechanisms can easily be carried out at the resource allocation level. This concept, originally proposed in the context of DVB-H [5], allows separating in time several fragments of the interleaved and coded data. These fragments, called slices of length $T_{\text {slic }}^{u}$, are spread over the radioframes according to a delay $T_{\text {slic }}^{\Delta}$ between each slice. Thereby, channel time diversity can be better exploited and the power consumption of the receiver can be lowered. This approach is depicted in Fig. 5 considering an enlarged TTI of $2 \mathrm{~ms}$, slices of length $1 \mathrm{~ms}$ and a delay of $5 \mathrm{~ms}$. Note that time slicing can lead to inter-frame interleaving as soon as the delay exceeds the radioframe duration. In that case, the data of a single $\mathrm{MCH}$ would be mapped onto several frames.

\section{Retransmission-based scheme}

Another way of taking advantage of time diversity is to exploit HARQ concepts already defined in the LTE unicast transmission mode. However, since no acknowledgment feedback signals are available in a broadcast mode, HARQ mechanisms have to be based on automatic retransmissions at the $\mathrm{MCH}$ level. A basic approach can consist in simply sending the same coded bits at each retransmission. In that case, a Chase combining process can be used at the receiver side. Such a scenario is illustrated in Fig. 6: in order to reach a code rate of $1 / 3$, three transmissions with code rate 1 can be used in three $1 \mathrm{~ms}$ subframes located in different frames.
The delay between retransmissions corresponds to the interleaving depth equal to $21 \mathrm{~ms}$ in this example. Note that this method avoids the enlargement of the TTI duration as well as the definition of an additional time interleaver.

A more elaborate technique could be based on an incremental redundancy mechanism: a given $\mathrm{CPHY}$ is broadcast during an initial subframe, and additional redundancy bits corresponding to the same information bits can be automatically retransmitted in subsequent subframes, after some predefined delays. Then, through the observation of the initial transmission content and of the additional redundancy, each receiver improves the decoding of the information.

Such an automatic retransmission approach results in a reduced code rate but yields increased diversity exploitation if the channel has sufficiently changed between two transmissions. It could only be implemented for push services, not for live TV or radio services, and in situation of limited data traffic, during the night for instance. A typical example of application would be data carousels, repeatedly delivering data in a continuous cycle during a sufficient period, allowing most of the receivers in the coverage area to retrieve the transmitted data.

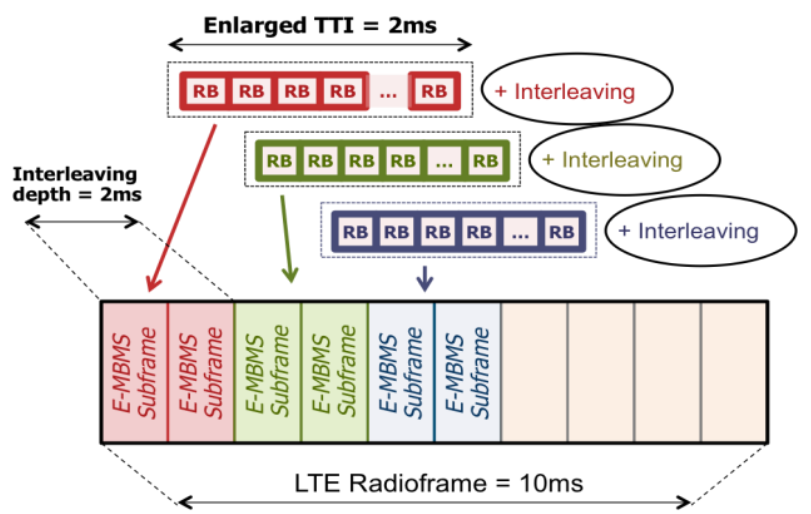

Fig. 4: Intra-frame interleaving and TTI enlargement of $2 \mathrm{~ms}$.

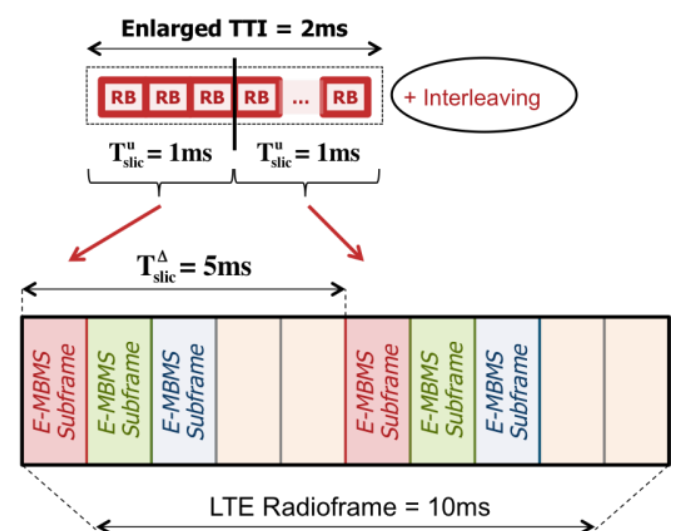

Fig. 5: Intra-frame interleaving combined with time slicing concept. TTI is enlarged to $2 \mathrm{~ms}$, slice duration is $1 \mathrm{~ms}$ and delay between slices is $5 \mathrm{~ms}$.

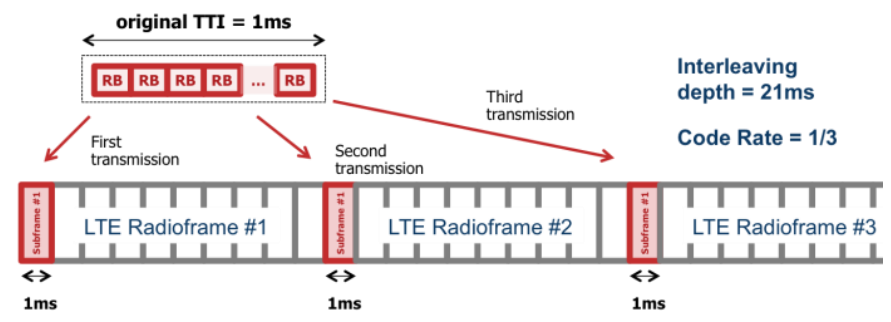

Fig. 6: Time interleaving based on automatic retransmissions 


\section{E. Sounding pattern design}

At this stage of the study, all the physical layer parameters of the proposed CPHY waveform have been identified and selected. Since the inter-carrier spacing values and the guard interval durations have been substantially modified, the Pilot Patterns (PP) specified by the LTE EMBMS standard are no more suitable. Indeed, scattered PPs can be viewed as a time-frequency sampling grid which granularity has to meet certain constraints to properly measure the time and frequency variations of the channel response. These constraints can be computed according to the Nyquist criterion (see [11], section 10.3.2.3.2 for more details):

$$
\begin{aligned}
& T_{N y q u i s t}=\max \left\{\frac{N_{F F T}}{F_{S}} \cdot \frac{X}{Y}, \frac{4}{3} \cdot G I\right\}, \\
& D_{N y q u i s t}=\frac{F_{S}}{2 N_{F F T} \cdot(1+G I) \cdot X},
\end{aligned}
$$

where $X$ denotes the spacing between 2 pilots in time domain and $Y$ denotes the spacing between 2 pilots in frequency domain. A convenient PP should hence be designed such that $T_{\text {Nyquist }}$ and $D_{\text {Nyquist }}$ remain greater than the guard interval duration and the maximum Doppler shift, respectively.

From these relations, it is then possible to propose PPs suitable for the different parameters of the CPHY, namely considering the inter-carrier spacing and guard interval values introduced in Table IV. In order to remain as close as possible to the LTE specifications, such PPs could be obtained following the same pattern shape as the one used in the LTE standard. However, since the PPs have to be unchanged from one TTI to another, such a strategy would lead to a dramatic increase of the overhead when the inter-
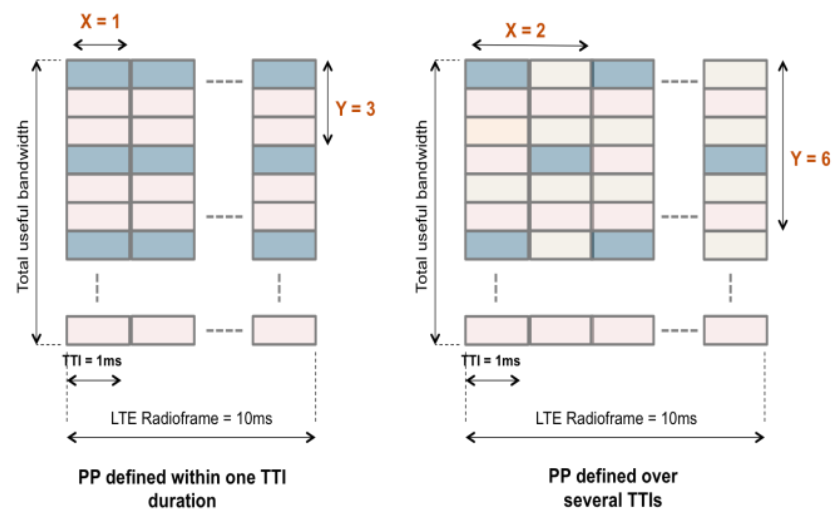

Fig. 7: Comparison of PPs defined in the $1.25 \mathrm{kHz}$ inter-carrier spacing case. On the left, the PP is restricted to the TTI time spread whereas it is defined over 2 TTIs on the right.

carrier spacing is reduced (see [12] for more details). Relaxing this constraint, i.e., allowing defining a PP over several TTIs, leads to more efficient PPs. As an example, Fig. 7 compares two suitable PPs with respect to the Nyquist criterion for the $1.25 \mathrm{kHz}$ inter-carrier spacing configuration. The first PP (on the left) strictly fulfils the LTE requirements by constraining the pattern to be entirely defined within a single TTI duration. The second PP (on the right) is built over two consecutive TTIs, thus leading to an overhead reduction by a factor of two. Note that the second approach would be compatible and particularly adequate in the case of an enlarged TTI, as proposed in section III.D for time interleaving improvement.

\section{F. Impact on terminals}

The resulting impact of $\mathrm{CPHY}$ implementation in receivers in terms of complexity and power consumption highly depends on the way the proposed generic CPHY is deployed and used. In the case it is deployed by a mobile or broadcast network operator on a dedicated carrier, a specific chipset is necessary to decode it. Conversely, in the case the CPHY is used in a mixed mode by a broadcast network operator (e.g. embedding CPHY frames in Future Extension Frames of DVB-T2) or by a mobile network operator (e.g. a time multiplex of LTE-Advanced/5G frames and CPHY frames in a shared-carrier mode), the addition of the new broadcast capabilities requires a multi-mode receiver whose complexity can be higher than the basic receiver. However, in the case of a time multiplex of LTE-Advanced and CPHY in a shared-carrier mode, the corresponding receiver should not differ very much from existing LTE-Advanced receivers with E-MBMS capabilities, due to the numerous commonalities between the proposed CPHY and E-MBMS.

\section{VERIFICATION AND IMPLEMENTATION OF THE CPHY CONCEPT}

Beyond the analysis and the specifications proposed in the previous sections, the validity of the CPHY concept was proved by means of simulations and implementation of a real time demonstrator.

Simulations were mainly carried out to assess the gains obtained when increasing the time interleaving depth according to section III.D, for both terrestrial and satellite channels. Besides, the demonstrator was built in two phases and was assigned two main objectives:

- Implementing a stand-alone CPHY transmission in order to assess the CPHY concept in transmissions over real Radio Frequency (RF) channels;

- Implementing CPHY and DVB-T2 combined together, in order to illustrate the feasibility of converged operation of the proposed CPHY and of a DVB-T2 signal in a single RF channel.

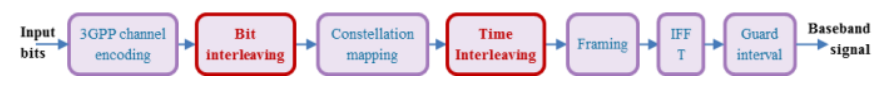

Fig. 8: Introduction of new interleaving blocks (in red) into the LTE transmission chain (terrestrial link).

\section{A. Validation of the CPHY concept}

\section{Validation of CPHY time interleaving enhancements}

Due to strong low-latency requirements, the LTE physical layer does not include any time interleaver. Nevertheless, as explained in section III.D such an interleaver can profitably be inserted into the CPHY in order to take advantage of the time diversity offered by the fading environment.

Therefore, for terrestrial transmission channels, a time interleaver coupled with a bit interleaver was introduced at the output of the coding and rate matching modules of the LTE transmission chain, as illustrated in Fig. 8. The simulated time interleaver structure is based on random interleaving combined with time slicing as proposed in Section III.D and illustrated in Fig. 9. In this way, the set of 
bits interleaved together are split into two slices of duration $T_{\text {slic }}^{u}$ and separated in time by the time slicing shift $T_{\text {slic }}^{\Delta}$. The bit interleaver depth is then equal to $2 T_{\text {slic }}^{u}$ while time diversity spans on $T_{\text {slic }}^{\Delta}+T_{\text {slic }}^{u}$.

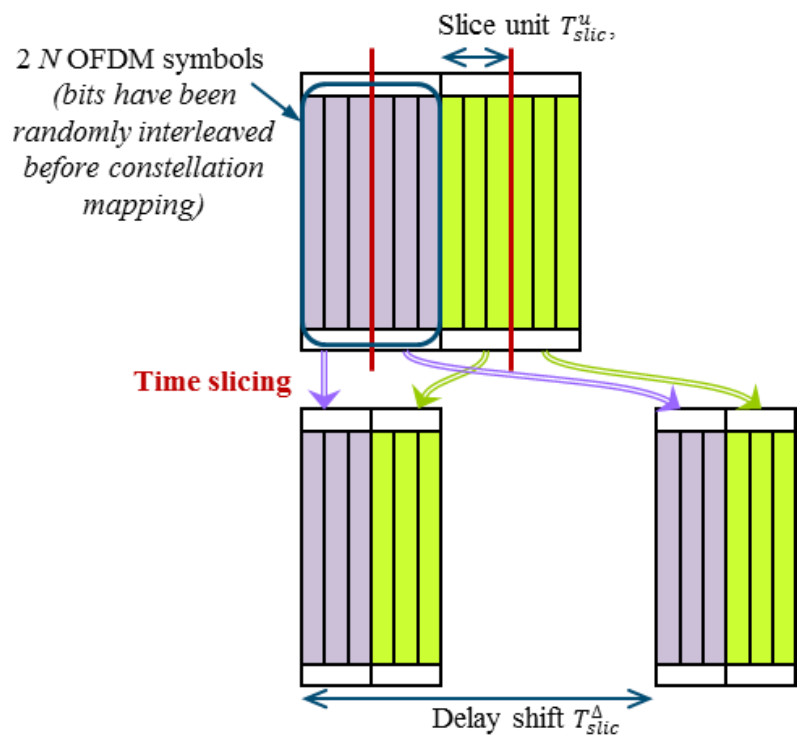

Fig. 9: Proposed time slicing principle (terrestrial link).

The impact of the time slicing algorithm on performance was assessed through simulations for the CPHY with a carrier frequency of $800 \mathrm{MHz}$ and with the intercarrier spacing set to $1.25 \mathrm{kHz}$ as proposed in Table IV. QPSK, 16QAM, and 64QAM constellations were considered with coding rates $1 / 2$ and $2 / 3$. Two different time slicing configurations corresponding to different values of slice duration $T_{\text {slic }}^{u}$ and time slicing shift $T_{\text {slic }}^{\Delta}$ were tested:

- $\quad T_{\text {slic }}^{u}=1 \mathrm{~ms}$ and $T_{\text {slic }}^{\Delta}=10 \mathrm{~ms}$,

- $\quad T_{\text {slic }}^{u}=2 \mathrm{~ms}$ and $T_{\text {slic }}^{\Delta}=20 \mathrm{~ms}$.

The channel model is the normalized typical urban mobile channel with 6 paths (TU-6) described in the DVBT2 implementation guidelines [11]. The mobile velocity is equal to $36 \mathrm{~km} / \mathrm{h}$, corresponding to a Doppler shift of 26.67 $\mathrm{Hz}$.

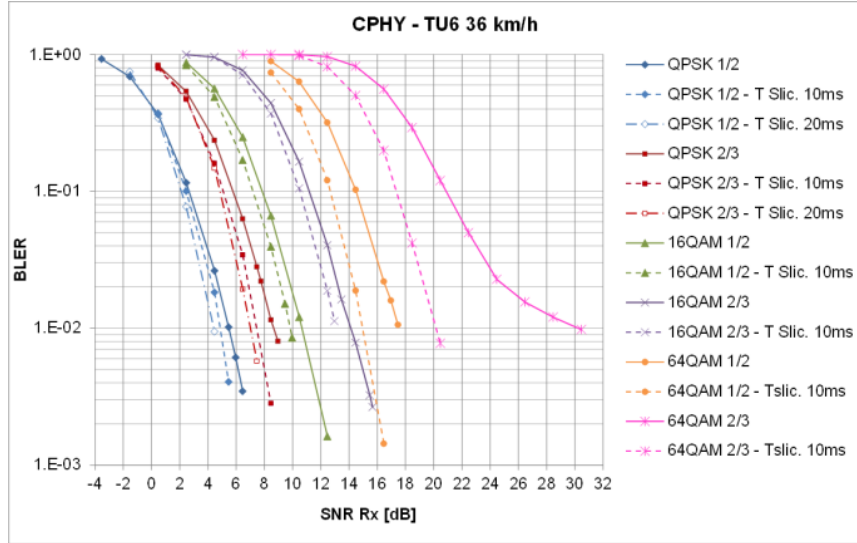

Fig. 10: Simulation results for the CPHY scenario of Table IV with and without time interleaving.

Fig. 10 shows the Block Error Rate (BLER) curves versus Signal to Noise Ratio (SNR) at the receiver side for the different simulated modulation and coding parameters, with and without time slicing. In the area of interest for broadcasting, that is for target BLERs lower than $10^{-2}$, this very simple time slicing algorithm brings a significant gain, ranging from $1 \mathrm{~dB}$ to $2 \mathrm{~dB}$, for most examples under study. In the particular case of 64QAM constellation with coding rate $2 / 3$, an error floor is observed when no time interleaving is implemented. This is due to the imperfect estimation of the Doppler shift performed by the channel equalizer, this effect being stronger for high order constellations. In this particular case, the simple introduction of the time interleaver significantly lowers the error floor so that a huge gain (around $10 \mathrm{~dB}$ ) can be observed in the $10^{-2}$ BLER region.

Performance enhancements due to time interleaving can also be demonstrated in satellite channels. Actually, satellite vehicular channels can suffer from fast fading events coupled with deep or average shadowing events whose correlation value can reach several meters [13]. Note that the CPHY waveform parameters in the satellite case can remain very close to those of the 3GPP LTE system, because of strong similarities in terms of frequency bands ( $5 \mathrm{MHz}$ bandwidth around $2 \mathrm{GHz}$ ) and owing to the fact that the coverage range is no more governed by the guard interval duration. Simulations have been performed with the LTE native intercarrier spacing, i.e., $15 \mathrm{kHz}$, and a guard interval of $1 / 14$, corresponding to a guard interval duration of $4.69 \mu \mathrm{s}$. The carrier frequency is set to $2.2 \mathrm{GHz}$. To address a very large scale of time interleaving values, simulations were obtained using a simple row-column matrix permutation.

Differently from the terrestrial channels, the BLER measurement is not currently used as a quality criterion for video reception over satellite mobile channels. In DVB-SH implementation guidelines [14], the Errored-Second-Ratio at $5 \%$ (ESR5) criterion is preferred to assess the transmission quality in mobile channels. In Land Mobile Satellite (LMS) channels, an ESR5 $90 \%$ fulfillment ratio is sought to ensure good video quality on a mobile device.

A first set of simulations were carried out in LMS with Intermediate Tree Shadowing (ITS) channel, with $40^{\circ}$ elevation for the satellite [13], and $60 \mathrm{~km} / \mathrm{h}$ mobile speed. The corresponding results are given in Fig. 11. One can observe that interleaving depths greater than or equal to 150 ms make the ESR5 $90 \%$ fulfillment ratio to be achieved at a minimum $\mathrm{C} / \mathrm{N}$ around $11 \mathrm{~dB}$. This $\mathrm{C} / \mathrm{N}$ value is quite achievable with a well-designed satellite payload and a vehicular category 1 terminal [14]. Significant interleaving gains exceeding $3 \mathrm{~dB}$ can be observed because of the severity of the considered LMS channel.

LMS ITS $60 \mathrm{~km} / \mathrm{h}$ QPSK $1 / 3$

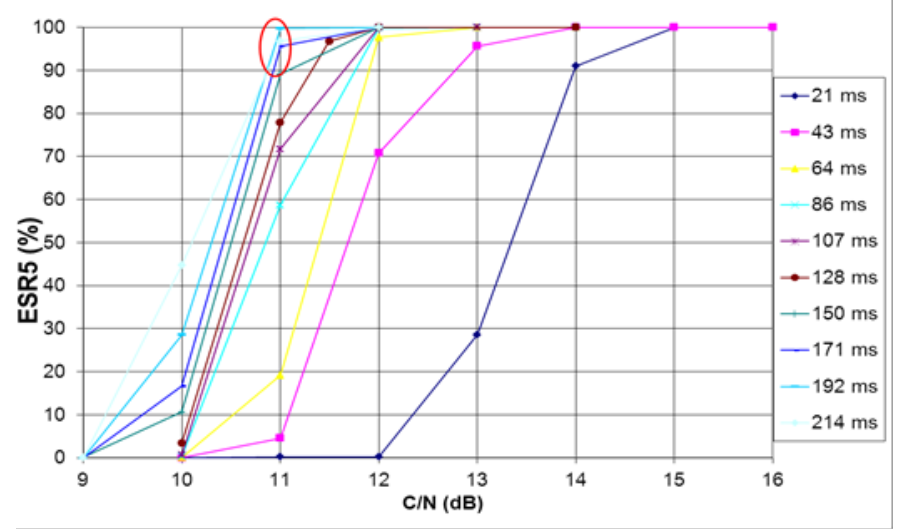

Fig. 11: Satellite case simulations results, LMS ITS channel. 


\section{CPHY demonstrator}

In order to validate the relevance of the CPHY specifications under realistic conditions, the CPHY system has been implemented in hardware using an FPGA platform. Fig. 12 depicts the overall architecture of the so-called Hardware Evaluation Platform (HEP) that is made of three entities, a main computing unit, the HEP Central Unit, and two secondary units, HEP Tx and HEP Rx.

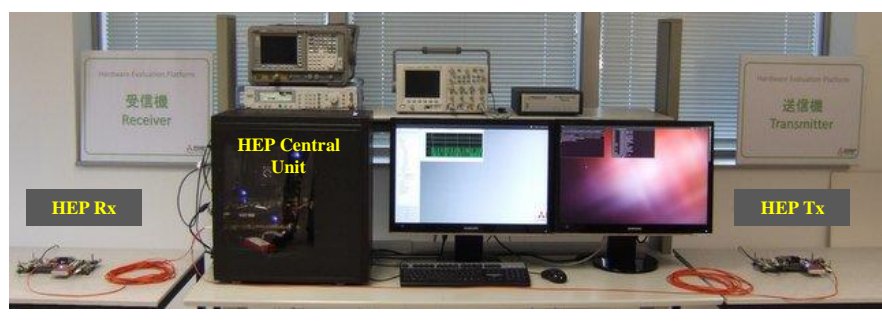

Fig. 12: Overview of the CPHY real-time implementation platform.

Both the transmitter and the receiver are implemented within the same equipment, namely the HEP Central Unit, which is made of a FPGA PCIe board embedded within a PC with a real-time operating system for control and monitoring. In order to allow for transmissions over distant locations, the main processing board is connected to the secondary units HEP Tx and HEP Rx via full-duplex optical links running at $5 \mathrm{Gbps}$. The HEP Tx unit implements the Digital-to-Analog Conversion (DAC) on an Intermediate Frequency (IF) while the HEP Rx unit implements the Analog-to-Digital Conversion (ADC) from IF down to baseband. The HEP Tx and Rx units can interconnect in analog with RF front-ends or with channel emulators, terrestrial or satellite. The CPHY platform is built from an existing design implementing a simplified version of the 3GPP LTE (Release 8) physical layer in downlink [15]. The reference platform was dedicated to the evaluation and the validation of physical layer technologies. There is thus no support of the signaling channels. Table $\mathrm{V}$ provides the key parameters of the reference 3GPP LTE system implemented in the platform.

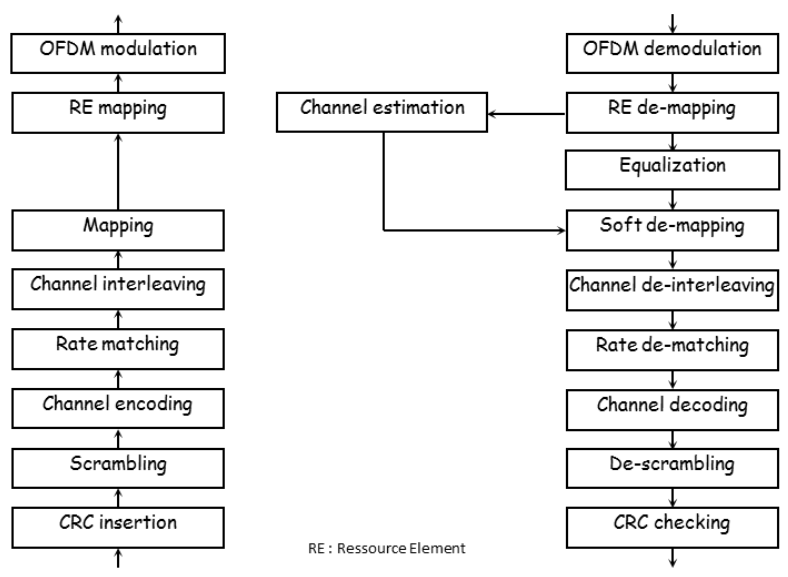

Fig. 13: Functional diagram of the CPHY platform.

Fig. 13 depicts the functional diagram of the CPHY transmitter and receiver in baseband. This version of the system does not support any time and frequency synchronization mechanism so far. The subset of CPHY specifications supported by the HEP platform is summarized in Table VI.
The main purpose of this first-generation demonstrator is to prove that the current 3GPP E-MBMS broadcasting component of the can easily be modified to enable broadcasting over large cells, as argued in section III.C. In the so-called Multicast-Broadcast Single Frequency Network (MBSFN) mode of E-MBMS, all the cells are tightly synchronized in time and transmit over the same frequency. MBSFN transmission enables highly efficient MBMS, allowing for over-the-air combining of multi-cell transmissions in the User Equipment (UE). An extended cyclic prefix is used to compensate for the difference in propagation delays, thus making the MBSFN transmission appear to the UE as a transmission from a single cell. The advantage of this SFN mode is precisely to allow for data transmission from a small number of very large cells.

The radius of the cells is actually limited by the duration of the guard interval of the OFDM symbols whose task is to avoid the inter-symbol interference due to long echoes coming from distant transmitters (see Fig. 14).

In order to demonstrate the gain provided by the CPHY system in terms of coverage area, the five different physical modes detailed in Table IV have been implemented for a $10 \mathrm{MHz}$ bandwidth. An essential guideline that directed the design of these physical modes was keeping maximum commonality with the 3GPP LTE system, especially the sampling frequency and the $1 \mathrm{~ms}$ duration of the 3GPP LTE TTI. With the normal 3GPP LTE subcarrier spacing mode and the SFN reduced subcarrier spacing modes, the system is able to transmit $12,6,3,2$ or 1 OFDM symbols per TTI as illustrated in Fig. 15. The most favorable case provides a guard interval of $200 \mu \mathrm{s}$, compatible with a coverage radius of $60 \mathrm{~km}$ (see Table IV). Note that the reduction of the subcarrier spacing also reduces the robustness to Doppler and that the selection of the mode to be deployed in practice depends on the operating frequency.

TABLE V

GENERAL PARAMETERS OF THE CPHY PLATFORM.

\begin{tabular}{lc}
\hline \hline & BASIC SYSTEM PARAMETERS \\
\hline \hline Carrier frequency & Low-IF $=70 \mathrm{MHz}$ \\
\hline Symbol duration & $66.66 \mu \mathrm{s}$ \\
\hline $\begin{array}{l}\text { Short cyclic prefix duration (symbols } \\
\text { 1-6 / 7) }\end{array}$ & \multicolumn{1}{l}{. } \\
\hline Total symbol duration & $71.35 \mu \mathrm{s}$ \\
\hline Subcarrier spacing & $15 \mathrm{kHz}$ \\
\hline Slot duration & $0.5 \mathrm{~ms}$ \\
\hline Number of symbols per slot & 7 \\
\hline Frame duration & $10 \mathrm{~ms}$ \\
\hline Number of subframes in a frame & 10 \\
\hline Number of subcarriers in a Resource & 12 \\
Block & SYSTEM PARAMETERS FOR 20 MHz CHANNELS \\
\hline \hline & $20 \mathrm{MHz}$ \\
\hline \hline Channelization bandwidth & $30.72 \mathrm{MHz}$ \\
\hline Sampling frequency & 15,360 \\
\hline Number of samples per slot & 2,048 \\
\hline FFT size & 848 \\
\hline Number of null subcarriers & 1,200 \\
\hline Number of modulated subcarriers & 100 \\
\hline Number of available RBs & \\
\hline
\end{tabular}


Trans. BROAdCASTING, SPECIAL ISSUE ON FoBTV

TABLE VI

CPHY FEATURES IMPLEMENTED IN HARDWARE

\begin{tabular}{cc}
\hline \hline Parameters & Supported values \\
\hline Bandwidth & $6,7,8$ and $10 \mathrm{MHz}$ \\
\hline Inter-carrier spacing & $1.25,2.5,3.75,7.5$ and $15 \mathrm{kHz}$ \\
\hline FFT size & $0.5 \mathrm{k}, 1 \mathrm{k}, 2 \mathrm{k}, 4 \mathrm{k}, 8 \mathrm{k}, 12 \mathrm{k}$ and $24 \mathrm{k}$ \\
\hline Pilot patterns & $\mathrm{X}=2,6$ \\
& $\mathrm{Y}=1,2,4$ and 6 (see section III.E) \\
\hline
\end{tabular}

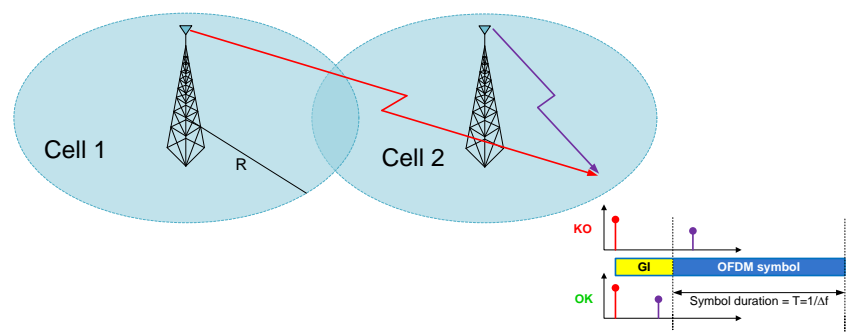

Fig. 14: Illustration of the impact of the distance between SFN transmitters on OFDM.

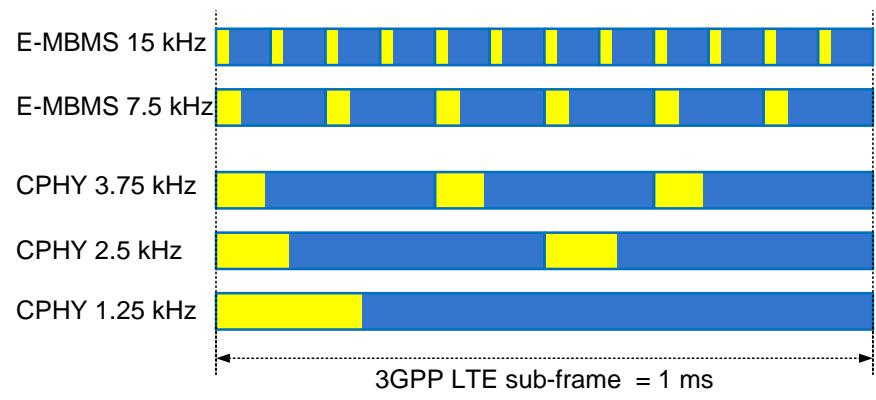

Fig. 15: Illustration of the guard interval duration with respect to the CPHY modes.

\section{Validation tests carried out with the CPHY demonstrator}

The five framing modes of the CPHY specifications have been implemented on the HEP platform with the ability to dynamically switch from one mode to another. Fig. 16 and Fig. 17 provide an overview of the HEP monitoring interface where the platform transmits an E-MBMS signal through a hardware channel emulator. In this particular case, the interface shows:

- at the transmitter side, the $3 \mathrm{D}$ representation of the time and frequency samples in the framing module and the transmitted signal in the time domain;

- at the receiver side, the $3 \mathrm{D}$ representation of the channel estimation samples and the constellation points at the output of the equalizer.

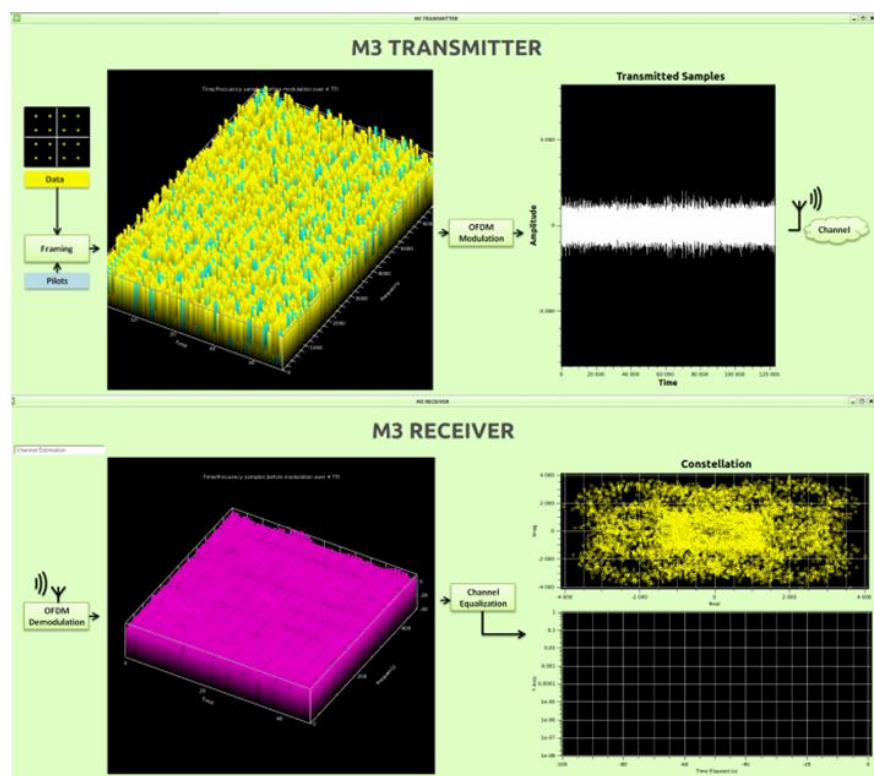

Fig. 16: Impact of a two-path channel with $160 \mu$ s delay on E-MBMS with $\Delta f=15 \mathrm{kHz}$.

To demonstrate the robustness of the CPHY system towards long echoes, a simple channel featuring two paths $160 \mu \mathrm{s}$ apart has been implemented. Fig. 16 illustrates the impact of this channel on the E-MBMS signal when the subcarrier spacing of $15 \mathrm{kHz}$ sets the guard interval duration to $16.7 \mu \mathrm{s}$. The signal is obviously degraded due to intersymbol interference. Once the platform configured to transmit the CPHY signal with the lowest subcarrier spacing value $\Delta f=1.25 \mathrm{kHz}$, the guard interval of $200 \mu \mathrm{s}$ is large enough to handle the channel delay and inter-symbol interference is no longer observed at the receiver side, as shown in Fig. 17.

\section{B. Embedding a CPHY waveform into DVB-T2 FEF frames}

The second phase of the demonstrator implementation combines two end-to-end real time transmission chains:

- The CPHY chain, including transmitter, channel emulator, and receiver, as described in the previous subsection;

- A DVB-T2 transmission chain also including transmitter, channel emulator, and receiver.

As illustrated in Fig. 18, the digital output of the CPHY modulator feeds the DVB-T2 modulator, which inserts the CPHY frames into reserved slots of the T2 signal, by means of the FEF mechanism offered by the DVB-T2 standard The resulting signal is a sequence of DVB-T2 and CPHY 


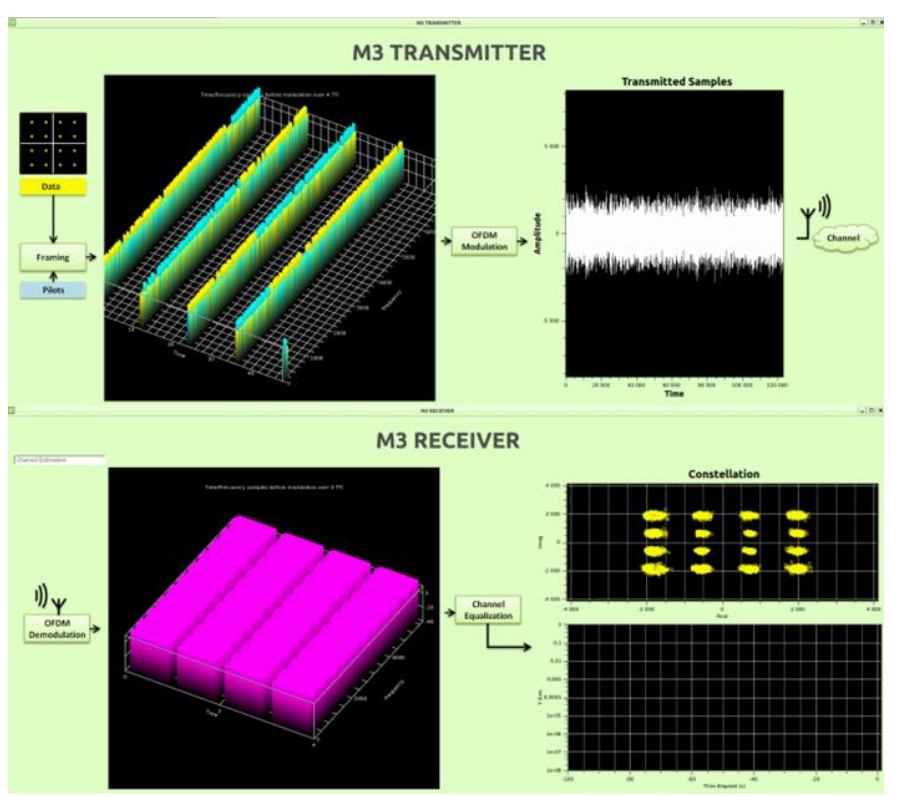

Fig. 17: Impact of a two-path channel with $160 \mu$ s delay on CPHY with $\Delta f=1.25 \mathrm{kHz}$.

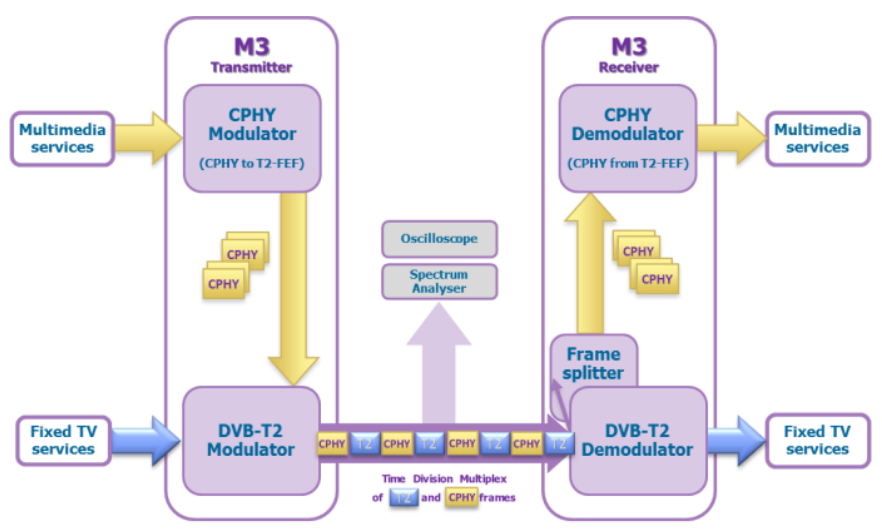

Fig. 18: Functional description of the demonstrator combining CPHY and DVB-T2 waveforms.

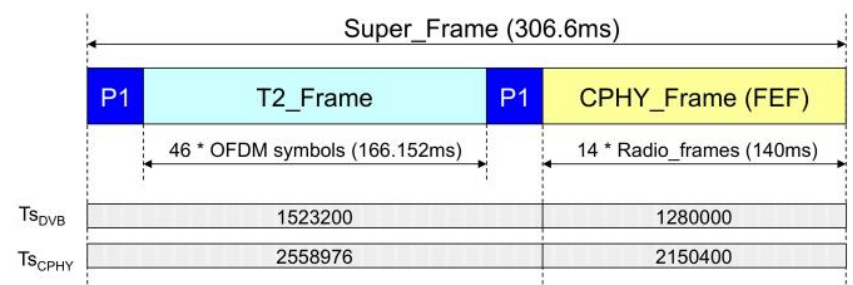

Fig. 19: T2 and CPHY Frame combination.

frames in a classical time division multiplexing scheme. At the receiver side, the DVB-T2 demodulator first provides channel estimation and overall synchronization, then extracts the CPHY frames and forwards them to the CPHY demodulator. On the one hand, the DVB-T2 frames are demodulated by the DVB-T2 demodulator which delivers the data to a standard audio/video (AV) decoder and a wide screen. On the other hand, the CPHY frames are demodulated by the CPHY demodulator which delivers the received data to the upper layers for display. Both demodulators provide indications about the quality of the received signals.
The structure of the DVB-T2 super-frame structure adopted for the combination of regular T2 frames and CPHY frames is given in Fig. 19 and the parameters of the T2 and CPHY TABLE VII

SUPER-FRAME PARAMETERS OF THE DVB-T2/CPHY PLATFORM.

\begin{tabular}{|c|c|}
\hline Frame format & 1 T2 frame + 1 FEF \\
\hline Bandwidth & $8 \mathrm{MHz}$ \\
\hline \multicolumn{2}{|c|}{ T2 FRAME PARAMETERS } \\
\hline DVB-T2 mode & Mode A (1PLP) - SISO \\
\hline $\mathbf{T s}_{\text {DVB }}$ & $7 / 64 \mu \mathrm{s}(109 \mathrm{~ns})$ \\
\hline \# OFDM symbols in T2 frame & 46 \\
\hline FFT size & $32 \mathrm{~K}$ extended $(27,841)$ \\
\hline$\Delta \mathrm{F}(\mathrm{kHz})$ & 0.279 \\
\hline Guard interval duration & $1 / 128(28 \mu \mathrm{s})$ \\
\hline Pilot pattern & PP7 \\
\hline PAPR reduction & No \\
\hline L1 constellation & 64QAM \\
\hline LDPC codeword length & $64 \mathrm{~K}$ \\
\hline Code rate & $2 / 3$ \\
\hline Constellation & Rotated 256QAM \\
\hline Time interleaver & intra-frame \\
\hline High efficiency mode & Yes \\
\hline T2 bitrate (Mbit/s) & 21.69 \\
\hline \multicolumn{2}{|c|}{ CPHY FRAME PARAMETERS } \\
\hline 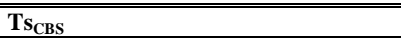 & $1 / 30.72 \mu \mathrm{s}(32.5 \mathrm{~ns})$ \\
\hline \# Radio frames in CPHY FEF (10ms) & 14 \\
\hline FFT size & $12 \mathrm{k}$ \\
\hline$\Delta \mathrm{F}(\mathrm{kHz})$ & 2.5 \\
\hline Guard interval & $1 / 4(33.33 \mu \mathrm{s})$ \\
\hline \# RBs & 40 \\
\hline \# Occupied subcarriers & 2880 \\
\hline Effective BW (MHz) & 7.2 \\
\hline TTI duration & $1 \mathrm{~ms}$ \\
\hline \# OFDM symbols per TTI & 2 \\
\hline \# OFDM symbols in CPHY frame & 280 \\
\hline Pilot pattern & (a) \\
\hline Constellation & 16QAM \\
\hline Code rate & $1 / 2$ \\
\hline CPHY bitrate (Mbit/s) & $\sim 3.5$ \\
\hline
\end{tabular}

frames are detailed in Table VII. The super-frame duration has been chosen to contain an integer multiple of both the DVB-T2 and CPHY elementary periods, $T s_{D V B}$ and $T s_{C P H Y}$. The configuration of the $\mathrm{T} 2$ frame is derived from the regular "UK mode", i.e., it targets fixed receivers at home, with wide high definition (HD) screens. Moreover, the super-frame structure was defined to have approximately the same duration for both the T2 and CPHY frames.

\section{Validation tests for a combined DVB-T2/CPHY transmission}

Three types of validation tests have been defined and carried out so far in order to show that CPHY frames can actually be transmitted within a T2 signal without disturbing the regular $\mathrm{T} 2$ reception:

- Transmitter test: verify with a spectrum analyzer and an oscilloscope the correct insertion of the CPHY-FEF into the T2 frame. When the CPHY signal is not present, the RF signal contains a null FEF corresponding to the CPHY-FEF duration. When the CPHY signal is present, the transmitter generates a continuous RF signal, as shown in Fig. 20 (the power for the CPHY and DVB-T2 signals have intentionally been set to different values in order to distinguish the FEFs).

- End-to-end go/no-go test (video test): check the video on the T2 receiver and on the CPHY receiver with cable transmission and over-the-air transmission via aerials. This scenario actually constitutes the convergence demonstration.

- Test in Additive White Gaussian Noise (AWGN) channel: perform a rough assessment of system 
performance, in presence of noise, for the transmission of the DVB-T2 and CPHY signals. The validation criterion is a video without error. The minimum carrierto-noise ratio requested for good reception is then compared to the values obtained by simulation.

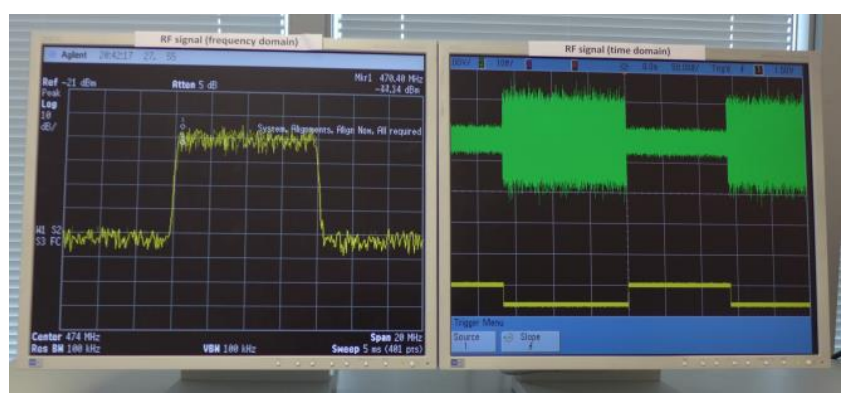

Fig. 20: Observation of a combined T2 and CPHY signal.

The complete assembled "CPHY in T2" demonstrator used to carry on these tests is shown in Fig. 21 and Fig. 22.

\section{Future work}

The first-generation demonstrators presented in this paper have been used to demonstrate both the CPHY concept and a possible broadband/broadcast convergent scenario with the insertion of CPHY traffic within DVB-T2

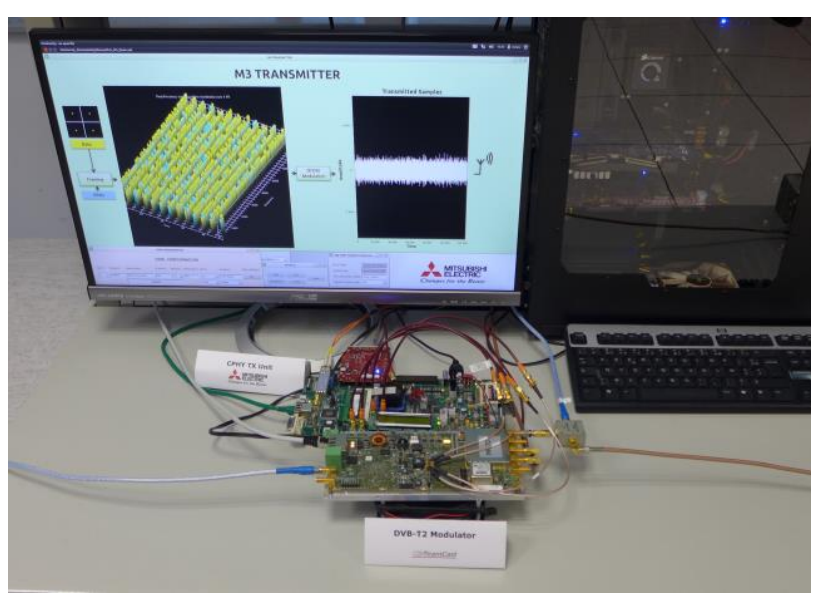

Fig. 22: (a) The "CPHY in T2" transmitter: The DVB-T2 modulator (front) and the CPHY Tx interface board (rear), the CPHY transmitter control display.
FEFs. However, only a limited set of the CPHY system parameters have been implemented and tested so far. The next tasks will involve implementing the missing modes in the CPHY demonstrator in order to achieve performance assessment.

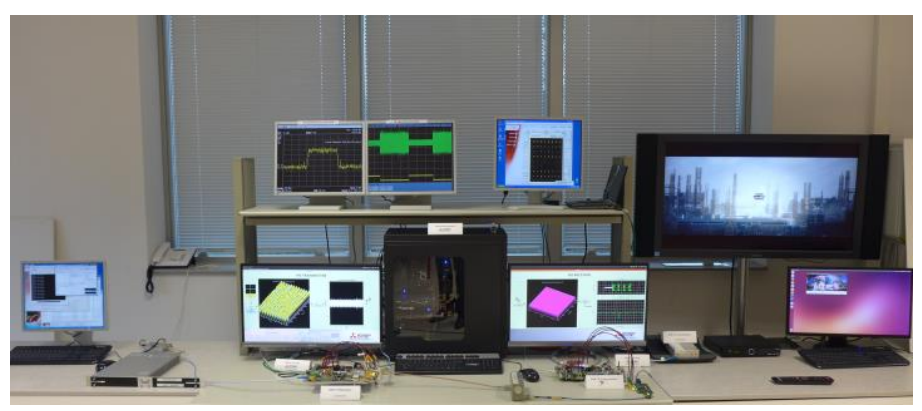

Fig. 21: The "CPHY in T2" overall platform: (from left to right) the DVB-T2 and CPHY video sources, the DVB-T2 and CPHY transmitters, the DVB-T2 frame splitter and CPHY receiver, the CPHY receiver control displays, the DVB-T2 receiver control display, the HD widescreen for DVB-T2 video and the screen for the low-resolution CPHY video.

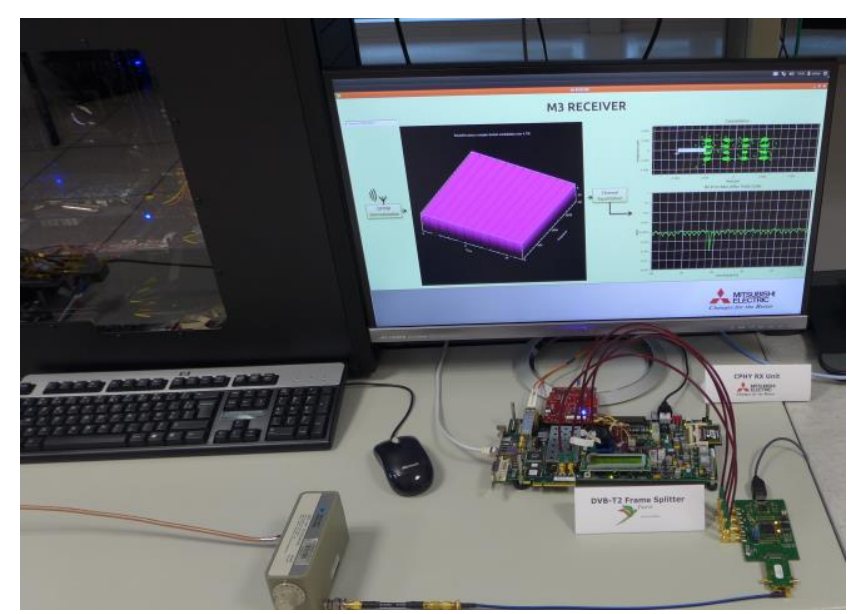

(b) The "CPHY in T2" receiver: The DVB-T2 frame splitter (front), the CPHY Rx interface board (rear), the CPHY receiver control display. 


\section{CONCLUSION}

This paper argues in favor of a broadband-broadcast convergence through the concept of common physical layer, providing a good basis for coverage cooperation between broadcast and mobile networks, while relying on nondedicated mobile receivers.

General technical requirements for the design of such specifications have been given and a practical example of a common physical layer based on the 3GPP LTE/E-MBMS and DVB-T2 standards has been described. Then, the successive steps of the proof of concept demonstration of the proposed unified broadcast layer have been presented: simulation results have proved the advantages of introducing time interleaving into 3GPP E-MBMS; the implementation of a hardware platform and subsequent validation tests have demonstrated the common physical layer feasibility as well as its embedding into a DVB-T2 future extension frame (FEF).

Additional laboratory tests with terrestrial/satellite hardware channel emulators and field trials have still to be carried out in order to complete the overall proof of concept demonstration. Nevertheless, the work already done has proved the technical feasibility and the relevance of such a common physical layer approach. Such a convergent approach appears to be a relevant work item in FoBTV's work on the next generation terrestrial broadcast systems.

The remaining issues mainly concern upper layers, signaling and, beyond physical layer, the idea of cooperation/convergence at the network architecture level has still to be considered. In particular, some kind of synchronization would be required between both networks and network infrastructures should be modified in order to create interconnections and insert gateways.

\section{ACKNOWLEDGEMENT}

The authors would like to warmly thank all the Mobile MultiMedia $\left(\mathrm{M}^{3}\right)$ project partners for the numerous and fruitful discussions that contributed to the maturation of the concepts outlined in this paper. Special thanks to the French National Research Agency for having funded this project in the framework of the VERSO research programme, which gave us the opportunity to undertake this study.

\section{REFERENCES}

[1] CISCO white paper, Cisco visual networking index: global mobile data traffic forecast update, 2012-2017, Feb. 2013,

http://www.cisco.com/en/US/solutions/collateral/ns341/ns525/ns537/ ns705/ns827/white_paper_c11-520862.pdf.

[2] http://www.ieee802.org/11/Reports/hew_update.htm

[3] http://www.nttdocomo.co.jp/english/binary/pdf/corporate/technology/ rd/technical_journal/bn/vol14_3/vol14_3_051en.pdf

[4] http://www.3gpp.org/Release-9

[5] Digital Video Broadcasting (DVB), Transmission system for handheld terminals (DVB-H), ETSI EN 302304 V1.1.1, Nov. 2004.

[6] http://en.wikipedia.org/wiki/MediaFLO

[7] Forward link only air interface; Specification for terrestrial mobile; Multimedia multicast, ETSI TS 102589 V1.1.1, Feb. 2009.

[8] Digital Video Broadcasting (DVB), Framing structure, channel coding and modulation for satellite services to handheld devices $(\mathrm{SH})$ below 3 GHz, ETSI EN 302 583, V1.1.1, March 2008.

[9] Digital Video Broadcasting (DVB); Frame structure channel coding and modulation for a second generation digital terrestrial
[10] Digital Video Broadcasting (DVB); Next generation broadcasting system to handheld, physical layer specification (DVB-NGH), DVB Bluebook A160, Nov. 2012.

[11] Digital video broadcasting (DVB); Implementation guidelines for a second generation digital terrestrial television broadcasting system (DVB-T2), ETSI TS 102 831, V1.2.1, Feb. 2012.

[12] C. Gallard et al., "Universal DVB-3GPP broadcast layer: an enabler for new business in mobile broadcasting landscape," Chapter 9 in Next generation mobile broadcasting, ed. D. Gómez-Barquero, CRC Press, 2013.

[13] F. Pérez-Fontán, M. Vázquez-Castro, C.E. Cabado, J. Pita García, and E. Kubista, "Statistical modelling of the LMS channel," IEEE Trans. Vehicular Technology, vol. 50, no.6, Nov. 2001, pp. 1549-1567.

[14] Digital Video Broadcasting (DVB); DVB-SH implementation guidelines, ETSI TS 102 584, V1.2.1, Jan. 2011.

[15] 3GPP TSG-RAN-E-UTRA, "3GPP TS 36.211, Physical Channels and Modulation (Release 8)”, v8.2.0 (2008-03).

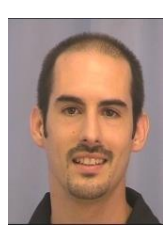

Matthieu Crussière received the M.Sc. and Ph.D. degrees in electrical engineering from the National Institute of Applied Sciences (INSA), Rennes, France, in 2002 and 2005, respectively. Since 2005, he has been an Associate Professor in the Department of Telecommunications and Electrical Engineering at INSA and currently leads his research activities at the Electronics and Telecommunications Institute of Rennes (IETR). His main research interests lie in digital communications and signal processing techniques. His works focus on the optimization of high-bit rate communications, resource allocation mechanisms and adaptive system design based on multi-carrier and multi-antenna schemes. He is author or co-author of more than 80 technical papers in international conferences and journals. He has been involved in several European and French national research projects in the field of powerline, broadcasting, ultra wideband and mobile radio communications.

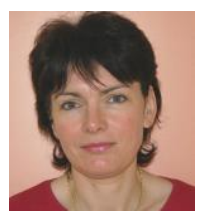

Catherine Douillard received the engineering degree in telecommunications from the Ecole Nationale Supérieure des Télécommunications de Bretagne, France, in 1988, the Ph.D. degree in electrica engineering from the University of Western Brittany, Brest, France, in 1992, and the accreditation to supervise research from the University of Southern Brittany, Lorient, France, in 2004. She is currently a Professor in the Electronics Department of Telecom Bretagne where she is in charge of the Algorithm-Silicon Interaction research team. Her main research interests are turbo codes and iterative decoding, iterative detection, the efficient combination of high spectral efficiency modulation and turbo coding schemes, diversity techniques and turbo processing for multi-carrier, multiantenna and multiple access transmission systems. In 2009, she received the SEE/IEEE Glavieux Award for her contribution to standards and related industrial impact.

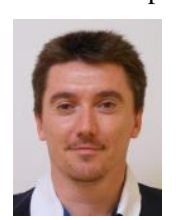

Christian Gallard received the engineering degree from the Ecole Supérieure d'Électronique de l'Ouest (ESEO), Angers, France, in 1996. From 1997 to 2000, he worked for Matra Communications in the area of digital communications applied to Professional Mobile Radio. From 2000 to 2002 he worked on digital communications in a UMTS context for Philips Consumer Communications (link layer simulations). From 2002 to 2004 he was a sub-contractor for France Telecom, working on speech processing, UMTS FDD and MIMO issues. He joined France Telecom in 2004. In 2005-2006, he worked on WiMAX issues (WiMAX Forum, link layer simulations). In 2006-2007 he was involved in elaboration of 3GPP LTE standard (RAN1 meetings, system definition, link layer simulations). Since the end of 2007, he has been involved in the definition of broadcasting systems (standardization of DVB-T2, DVB-NGH, B21C, ENGINES Celtic projects). From 2010 to 2013, he led the French collaborative M3 (Mobile Multi-Media) project. He is today a research project coordinator inside Orange (studies on enablers in digital communications, propagation channel modelling, antennas design.. for future networks, including $5 \mathrm{G}$ ).

Marie le Bot received the engineering degree from the Ecole Centrale de Nantes, France, in 1997; and the $\mathrm{PhD}$ degree in electronics and communications from the Conservatoire National des Arts et Métiers, Paris, France, in 2001. Since then, she has been working for Orange Labs, France, as a research engineer in digital signal processing, dimensioning and resource management of wireless networks (GSM, UMTS, HSPA, LTE) and power-line communications (PLC). Her current areas of research are the mobile broadcasting systems and the reduction of energy consumption in home networks. 


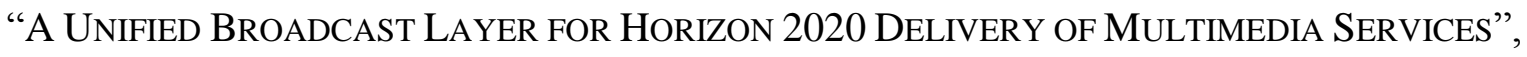

SUBMITTED TO IEEE TRANS. BROADCASTING, SPECIAL ISSUE ON FOBTV

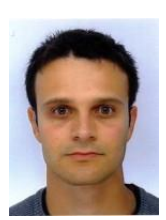

Benjamin Ros graduated from the University of Rennes1

in electronics and telecommunications engineering in 2005. He has first worked on signal processing algorithms for GNSS receivers. Since 2007, he has been working at the French Space Agency in the telecommunications department as an air interface engineer on topics such as waveforms optimization over satellite mobile channel, channel sounding, and satellite system optimization.

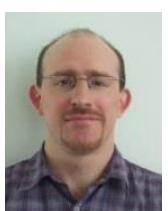

Arnaud Bouttier was born in Le Mans, France, in 1969. In 1992, he received a Master of Sciences on Signal Processing from the University of Rennes I. He graduated from the Engineering school Ecole Nationale Supérieure des Télécommunications de Bretagne (ENSTB) in 1994. $\mathrm{He}$ received the $\mathrm{Ph} . \mathrm{D}$. degree from the University of Rennes I in 2000. During his Ph.D., his research was focused on blind equalization by means of adaptive trellis filtering. $\mathrm{He}$ joined MERCE, formerly ITE-TCL, in 2000 with the task to acquire knowhow on digital implementation of telecommunication systems. He is currently project leader of the development of hardware evaluation platforms in the Wireless Communication Systems team with an emphasis on satellite and terrestrial broadcasting. He was involved in several collaborative projects dealing with broadband and broadcasting technologies (IST 4MORE, ANR APOGEE, CELTIC WINNER+, CELTIC ENGINES, ANR M3, etc.).

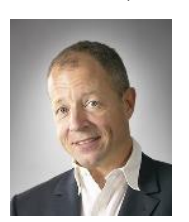

Alain Untersee received the engineering degree in electronics from the National Electronics Engineering School in Grenoble, France. After several years spent in large French groups, he co-founded in 1988 the ITIS Company (Innovation in Telecommunications, Image, and Sound) where he held the position of Sales Director. He developed the worldwide sales of professional equipment for Terrestrial Digital TV (DVB-T) and Digital Radio (DAB) networks. He joined TeamCast in 2007 where he is taking care of the involvement of TeamCast in collaborative research projects at the French and international levels (preparation of proposals, management of projects). 